\title{
A Perspective on Radar Remote Sensing of Soil Moisture
} Sang-Eun Park ${ }^{\dagger}$

Graduate School of Science and Technology, Niigata University, Niigata 950-2181, Japan.

\begin{abstract}
The sensitivity of microwave scattering to the dielectric properties and the geometric structure of soil surfaces makes radar remote sensing a challenge for a wide range of environmental issues directly related to the condition of natural surfaces. Especially, the potential for retrieving soil moisture with a high spatial and/or temporal resolution represents a significant contribution to hydrological and ecological modeling. This paper aims to review the current state of the art in SAR technology and methodological issues towards the discovery of a new potential accurate monitoring of soil moisture changes. In this paper, important parameters or constraints significantly affect the sensitivity of the measurements to soil moisture, such as roughness statistics, spatial resolution, and local topography, are discussed to improve the applicability of SAR remote sensing techniques. This study particularly intends to discuss important notes for developing smart and reliable methods capable of retrieving geophysical information.
\end{abstract}

Key Words : Microwave Remote Sensing, Synthetic Aperture Radar, Surface Scattering Model, Soil Moisture.

\section{Introduction}

The knowledge of soil moisture is important for hydrology, agronomy, meteorology, and numerous other Earth systems sciences. Ground observations cannot satisfy the data requirements of many applications, because in-situ measurements of the both spatially and temporally variable soil moisture field represent limited point measurements. Owing to the sensitivity of microwave scattering to the dielectric properties of the target, the potential for retrieving soil moisture has been recognized as one of the most important application of Synthetic Aperture Radar (SAR) remote sensing for several decades
(Beckmann and Spizzichino, 1963; Bass and Fuks, 1979; Ulaby et al., 1982; Ulaby and Elachi, 1990; Fung, 1994; Quesney et al., 2000; Wu et al., 2001; Glenn and Carr, 2003; Kim and van Zyl, 2009). Nonetheless, a retrieval of soil moisture at a scale and accuracy commensurate with the needs of environmental and commercial applications is still difficult because of the inherent complexities hitherto not yet fully understood.

Microwave-based retrieval of soil moisture has been limited to a certain degree by the natural variability and complexity. With rapid development of space technology in remote sensing, such as hardware, signal processing, and antenna design, it is

Received October 2, 2011; Revised October 29, 2011; Accepted November 1, 2011.

${ }^{\dagger}$ Corresponding Author: Sang-Eun Park (s.park@wave.ie.niigata-u.ac.jp) 
important to establish the utility of them for the operational observation of global environments including soil moisture. This study aims to review the current state of the art in methodological issues towards the discovery of a new potential accurate monitoring of soil moisture changes. In this paper, important parameters or constraints significantly affect the sensitivity of the measurements to soil moisture are discussed to improve the applicability of SAR remote sensing techniques.

\section{Surface Scattering Models}

The backscattering coefficient observed from radar sensors can be expressed as a function of the radar configuration (frequency, polarization, and incidence angle) and various surface geophysical parameters Therefore, the polarimetric backscattering coefficient $\sigma^{0}$ can be written as:

$$
\sigma_{p q, f, \theta}^{0}=F\left(x_{1}, x_{2}, \cdots, x_{N}\right),
$$

where the subscripts $p q, f$, "and" $\theta$ are polarization, frequency, and incidence angle of the transmitting and receiving microwave signal. Independent variables $x_{i}(i=1,2, \cdots, N)$ are surface geophysical parameters. For the bare soil case, these states variables can include the volumetric moisture content of top soil and statistical roughness parameters of the soil surface.

Various empirical and physical models have been developed to describe the forward relationship $F$ between $\sigma^{0}$ and surface geophysical parameters. One of the simplest expressions is linear model function (Ulaby et al., 1982; Quesney et al., 2000; Glenn and Carr, 2003), considering only the soil moisture contents, $m_{V}$, which can be written as:

$$
\sigma_{p q, f, \theta}^{0}=C m_{V}+D
$$

In most of previous studies, the slope (C) and the offset (D) of this linear function are estimated by regression analysis on the basis of in-situ measurements. It has been widely used mainly due to their simplicity. However, the regression relationship can be condition- specific and, thus, it is difficult to use it for radar signal inversion without timeconsuming calibration work. Recently, Kim and van Zyl (2009) proposed a new algorithm to determine these two unknowns based on time-series approach using radiometer and radar data. This time-series algorithm can be a promising approach, which provides a simple and robust way to estimate soil moisture.

On the other hand, theoretical relationships between backscatter coefficients and surface parameters have been developed. In the microwave region, common approximate methods for modeling rough surface scattering are the Small Perturbation Method (SPM) and the Kirchhoff Approach (KA) (Beckmann and Spizzichino, 1963; Bass and Fuks, 1979; Ulaby and Elachi, 1990; Fung, 1994; Wu et al., 2001). For example, the backscattering coefficients for surfaces with small roughness and short correlation lengths can be described by the SPM, such as:

$$
\sigma_{p p, f, \theta}^{0}=\frac{4}{\pi} k^{2} \cos ^{4} \theta\left|\alpha_{p p}\right|^{2} \mathrm{~W}(-2 k \sin \theta),
$$

where $k=2 \pi f / \mathrm{c}$ is the radar wavenumber, $\alpha_{p p}$ is Bragg scattering coefficients determined by the dielectric constant of the soil surface which is related to the soil moisture contents (Wang and Schmugge, 1980; Dobson et al., 1985). In addition, $W(K)$ is the surface roughness spectral density. The nonlinear forwards relationship $F$ between $\sigma^{0}$ and surface geophysical parameters includes a coupled effect of roughness parameters and moisture contents on the polarimetric backscatter.

In the development of a theoretical model for wave 
scattering from randomly rough surfaces, much effort has been devoted to broadening the range of validity over SPM and KA. Recently, a surface scattering model derived from the integral equation method (IEM) (Fung, 1994; Wu et al., 2001) has bridged the gap between these two models successfully. The horizontal and vertical polarized single backscattering coefficient from the IEM model is given by

$$
\sigma_{p p, f, \theta}^{0}=\frac{k^{2}}{4 \pi} k e^{-2(\mathrm{kscos} \theta)^{2}} \sum_{n=1}^{\infty}\left|\mathrm{I}_{p p}^{n}\right|^{2} \frac{W^{(n)}(-2 k \sin \theta)}{n !},
$$

where $\mathrm{I}_{p p}^{n}$ is a function determined by the dielectric constant of the soil surface (Fung, 1994; Wu et al., 2001), $s$ is the rms height of the roughness profile and the function, and $W^{(\mathrm{n})}(K)$ is $n^{\text {th }}$ power of the surface roughness spectral density. However, IEM is difficult to solve analytically because the geophysical parameters, such as surface roughness and dielectric constant, are functionally coupled. More details on difficulties in the microwave scattering model for the practical application to soil moisture retrieval are discussed in next Session.

\section{Issues in Scattering from Soil Surface}

One of the major disturbing factors in the estimation of the soil moisture contents from radar remote sensing is vegetation effects on radar backscattering coefficients. When vegetation scattering mechanism is dominant among the radar backscattered signals, e.g., scattering from densely forested areas, it is not possible to retrieve moisture contents of soil surface. If we have low vegetation cover, radar remote sensing has high potential to retrieve soil moisture contents due to the relatively long wavelength of the microwave signal. However, a good estimation of scattering and absorption phenomena in vegetation is still a difficult task. A lot of radar parameters, e.g., frequency and incidence angle, and bio-physical parameters, e.g., vegetation types, ages, and seasonal variability, are related to this issue. In this study, we restrict our attention to a bare soil case.

Even in case of the bare soil surface, modeling surface scattering has proven to be more difficult than originally anticipated (Ulaby, 1998). One of the main issues in quantitative estimation of soil moisture contents from radar data is to eliminate the confounding influence of surface roughness on it. That is, correct estimation of surface roughness is required in order to obtain soil moisture contents from SAR data.

\section{1) Roughness of Soil Surface}

The statistical variation of a random rough surface is characterized by its height distribution and surface correlation function. The height distribution of soil surfaces is usually assumed as the Gaussian function characterized by its rms height s, such as

$$
s=\sqrt{\left\langle\mathrm{Z}(\mathrm{x})^{2}\right\rangle-\langle\mathrm{Z}(\mathrm{x})\rangle^{2}},
$$

where $z(x)$ is the surface height profile and $<>$ denote ensemble average. For simplicity we only consider 1-D rough surface in this study. Since the profile usually measured from the reference mean line, the mean value of surface profile $\mu=\langle z(x)>$ is usually assumed as zero. On the other hand, the spatial correlation property of the surface can be described by the surface autocorrelation $R(l)$, such as:

$$
R(l)=<z(x) z(x+l)>.
$$

The surface correlation length $l_{c}$, which is defined as the displacement such that $R\left(l_{c}\right)$ is equal to $e^{-1}$, widely used as a means to express horizontal roughness of the surface.

In the surface scattering models, adequate mathematical expressions for the autocorrelation function $(\mathrm{ACF})$ of the target surface is one of the essential model parameter to obtain accurate 
backscattering simulations. The commonly used ACF is either Gaussian or Exponential function, given by:

$$
\begin{gathered}
R(l)_{G}=s^{2} \exp \left(-l^{2} / l_{c}^{2}\right) \text { and } \\
R(l)_{E}=s^{2} \exp \left(-|l| l_{c}\right),
\end{gathered}
$$

respectively. Then, the theoretical surface scattering models are formulated in terms of the surface roughness power density (PSD), which is the Fourier transform of the ACF. The Gaussian and Exponential PSD functions are given by:

$$
\begin{gathered}
W(K)_{G}=s^{2} \pi l_{c} \exp \left(-l_{c}^{2} K^{2} / 4\right) \text { and } \\
W(K)_{E}=s^{2} 2 l_{c}\left[1+l_{c}^{2} K^{2}\right]^{-1} .
\end{gathered}
$$

Fig. 1 shows examples of the Gaussian and the Exponential ACF and corresponding PSD with s =

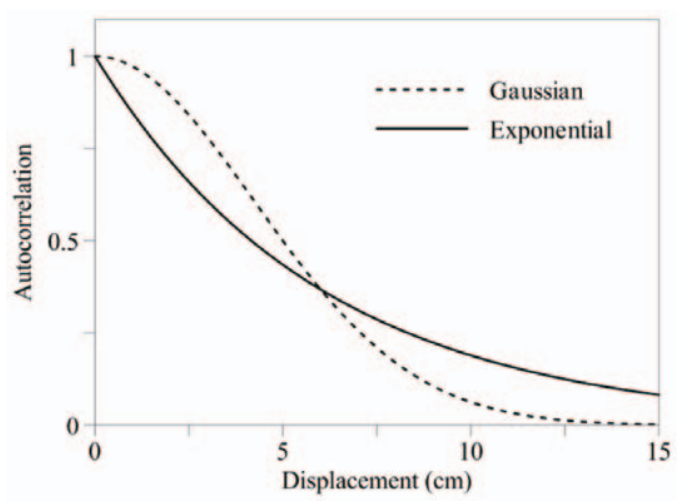

(a)

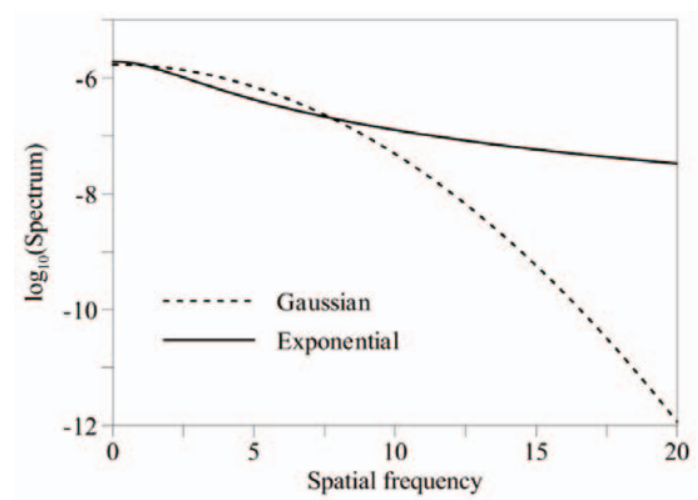

(b)

Fig. 1. An example of the Gaussian and Exponential (a) autocorrelation and (b) roughness power density functions.
$0.4 \mathrm{~cm}$ and $l_{c}=6 \mathrm{~cm}$. Although both functions have the same rms height and correlation length their forms are very different. The exponential function falls away faster from the origin, implying a more rapid loss of correlation and hence the presence of roughness on a fine scale. Consequently, the power spectrum of the exponential surfaces indicates the presence of high frequency, or short-wavelength, roughness components. The Gaussian function, however, falls away more slowly, leading to correlation on a longer scale.

\section{2) Roughness Effect on Backscattering Coefficients}

In practical application of the forward and inverse models of the radar signal, the theoretical scattering models usually assume one of the theoretical ACF to reduce the number of unknown and to obtain closed form expression. Fig. 2 illustrates the effect of roughness ACFs on the estimation of polarimetric backscattering coefficients. The simulation is performed at C-band frequency and the roughness parameters of $\mathrm{s}=0.4 \mathrm{~cm}$ and $l_{c}=6 \mathrm{~cm}$. One can find a large difference in angular trend between the exponential and the Gaussian ACF, particularly in the higher angular range. Fig. 3 shows a dependence of

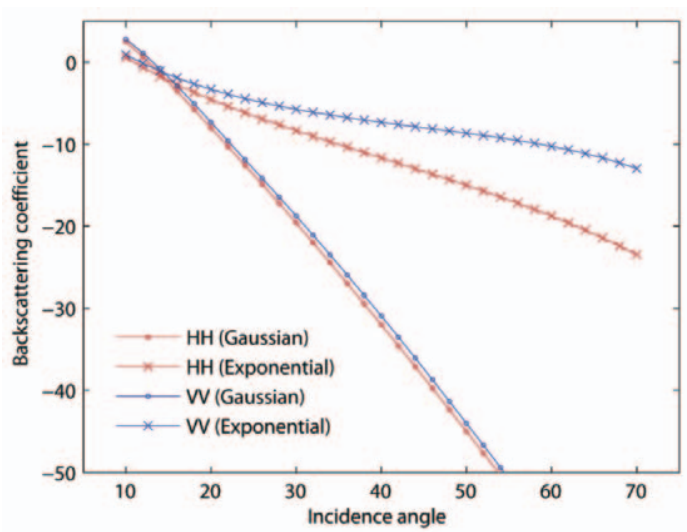

Fig. 2. The effect of the autocorrelation function on the angular response of the backscattering coefficients. 


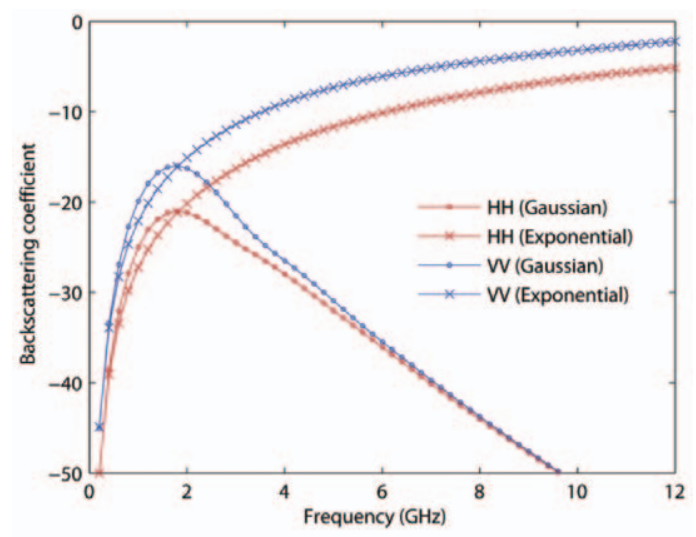

Fig. 3. The effect of the autocorrelation function on the backscattering coefficients plotted versus radar frequency.

backscattering coefficients on the radar frequency at $40^{\circ}$ of the incidence angel for the Gaussian and the Exponential ACF. The Exponential surface is characterized by short-range fluctuations, whereas the Gaussian surface appears smooth on this length scale. Consequently, the backscattering coefficients from two ACF show a large difference at higher frequency range.

Both Fig. 2 and Fig. 3 show the importance of the shape of the correlation function on the estimation of backscattering coefficient. It has been shown that the ACF from field measurements are complicated and usually vary between Gaussian and exponential functions (Oh et al., 1992; Davidson et al., 2000) resulting in difficulties in modeling backscatter signal and retrieving soil moisture contents. It seems that, in addition to the rms height and the correlation length, more states variables indicating the shape of ACF is required for the forward and inverse scattering models particularly in case of high incidence angle and high frequency.

\section{3) Roughness Parameterization}

For a large resolution cell size, the observed surface can be considered infinite and Eq. (6) can be used to estimate roughness correlation properties.

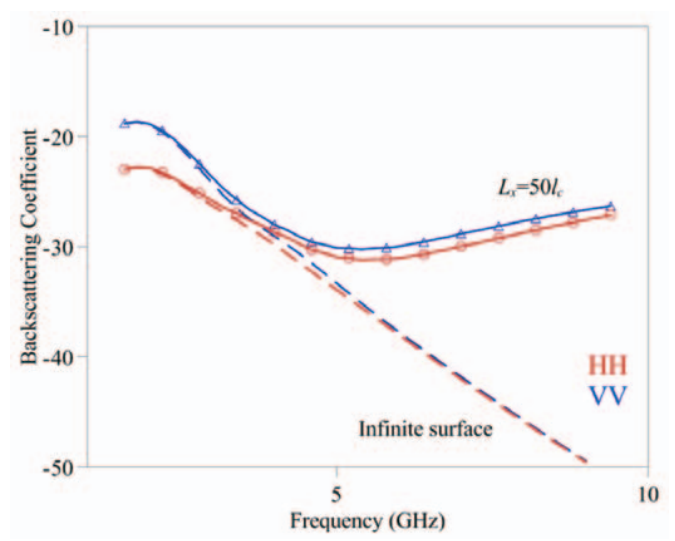

Fig. 4. The dependence of backscattering coefficient on the radar frequency at $40^{\circ}$ of incidence angel for different spatial resolution.

However, in practice, a radar image is acquired from a coherent summation of elementary contributions within finite ground resolution $L_{x}=\mathrm{c} /(2 B \sin \theta)$, where $\mathrm{c}$ denotes the speed of light, and $\mathrm{B}$ is the chirp bandwidth of the radar signal. For high resolution case, the autocovariance function of the truncated surface corresponding to the resolution cell, $L_{x}$, can be expressed as (Park et al., 2008):

$$
\rho(l)=\left(1-\frac{|l|}{L_{x}}\right)\{R(l)-\operatorname{var}(\bar{z})\},
$$

where $\operatorname{var}(\bar{z})$ is the variance of the sample means. Since the roughness profile for the resolution cell is truncated from infinite surface with rectangular window, $\rho(l)$, is defined in a constant range $L_{x}$ through the triangular function, $\left(1-|l| / L_{x}\right)$.

Fig. 4 shows the dependence of co-polarized backscattering coefficients on the radar frequency. The simulation is performed at $40^{\circ}$ of incidence with the Gaussian ACF of infinite surface and those of truncated surface. The use of conventional Gaussian ACF based on the infinite surface into scattering model can provide an underestimation of the backscatter particularly at high frequency range. Consequently, the roughness properties of the truncated surface should also be carefully considered in the application of imaging radar to soil surfaces. 


\section{4) Effect of topography}

When a topographic variation exist inside a resolution cell, e.g., row structure of agricultural fields, it can be considered as a large scale roughness component. In this case, it is necessary to separate the small scale roughness (random component) that influences the backscattering process discussed above from large scale (low frequency) periodic pattern. Then, one can implement alternative scattering models, such as the two-scale model which generally result from a cut of the roughness spectrum at boundary wavenumber (Lemaire et al., 2002).

When radar images rugged terrain whose scale is larger than the resolution cell, however, it becomes necessary to consider topography-induced changes of the backscatter. The local incidence angle, which is defined as the angle between the line of sight direction and the surface normal, should supersede the incidence angle in Eq. (3) and (4). In addition, the local slope induces the shifts in the polarization orientation angle. The amount of topographical slopeinduced shift in the polarization orientation angle can be considered as the rotation of the ground scattering matrix about the line of sight (Lee et al., 2002):

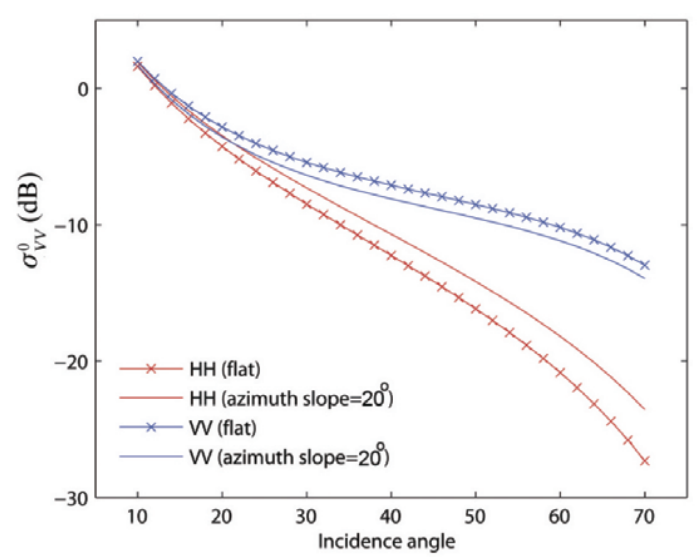

Fig. 5. The effect of terrain slope on the angular response of the backscattering coefficients.
$\left[\begin{array}{ll}S_{H H} S_{H V} \\ S_{V H} & S_{W}\end{array}\right] \rightarrow\left[\begin{array}{cc}\cos \psi & \sin \psi \\ -\sin \psi & \cos \psi\end{array}\right]\left[\begin{array}{l}S_{H H} S_{H V} \\ S_{V H} S_{W}\end{array}\right]\left[\begin{array}{cc}\cos \psi & -\sin \psi \\ \sin \psi & \cos \psi\end{array}\right]$.

Fig. 5 shows an example of the effect of topography on the estimation of surface backscatter. It should be noted that the backscattering coefficients which have been used for the study of surface hydrology are not rotational-invariant and, thus, the estimation of them from forward and inverse scattering models can be biased due to the presence of large scale topography.

\section{Discussion on Soil Moisture Retrieval Methods}

The general objective radar remote sensing for natural surface is to find the inverse mapping of the problem given in Eq (1). As we discussed above, it contains several states variables to be estimated. Even if we can assume the simplest expression of the forward relationship between radar measurements and surface geophysical parameters, three unknowns must be determined. Therefore, conventional single polarization, single frequency, and single pass radar remote sensing cannot correctly address this problem without a priori information. To obtain reliable information on surface properties from radar observations, it is necessary to avoid such an underdetermined problem in the inversion process. One possible way to deal with the inversion problem is to reduce the number of unknown surface variables. For instance, studies have researched a relation between rms height and correlation length, such as a powertype relation in (Baghdadi et al., 2004), to reduce the number of roughness parameters. The other way is to increase the number of independent radar measurements. Various approaches have been investigated including multi- incidence angle, multi- 
frequency, multi-temporal, and fully polarimetric approaches.

\section{1) Multi-incidence approach}

The difference in backscatter generated by the scattering models with two different incidence angles, keeping all other parameters constant, is only proportional to roughness parameters such as:

$$
\Delta \sigma_{p q}^{0}=\sigma_{p q}^{0}\left(\theta_{1}\right)-\sigma_{p q}^{0}\left(\theta_{2}\right)=f\left(s^{2} / l_{c}\right) .
$$

Studies have reported that it is possible to obtain both soil moisture contents and roughness parameters from two radar images with different incidence angle and one image with dry condition (Zribi and Dechambre, 2002; Rahman et al., 2008). This approach requires that the dielectric and geometric properties should remain constant during the multiangular acquisitions. Therefore, this approach is can hardly applicable to operational remote sensing of soil moisture because current radar systems cannot acquire multi-angular imagery during a single overpass.

\section{2) Multi-frequency approach}

The number of radar measurements can be increased by multi- frequency radar acquisition. Both roughness parameters and soil moisture contents can be estimated by L-, C-, and X-bands data acquired simultaneously (Bindlish and Barros, 2000; Gade et al., 2008) such as:

$$
\left[\begin{array}{c}
m_{V} \\
s \\
l_{c}
\end{array}\right]=F^{-1}\left(\left[\begin{array}{c}
\sigma_{V V, \mathrm{~L}-\text { band }}^{0} \\
\sigma_{V V, \mathrm{C}-\text { band }}^{0} \\
\sigma_{V V, \mathrm{X} \text {-band }}^{0}
\end{array}\right]\right) \text {. }
$$

However, similar to the multiple incidence angle approach, it has an inherent limitation in operational application due to a high temporal variety of surface geophysical parameters and a rare availability of multi- frequency SAR data acquired simultaneously.

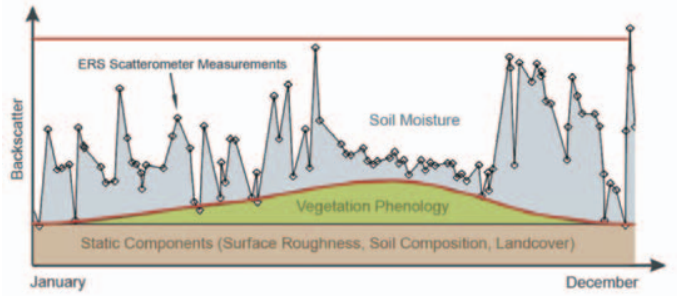

Fig. 6. Schematic diagram of a soil moisture retrieval algorithm based on the change detection approach (Courtesy from Wolfgang Wagner, TU-Wien).

\section{3) Multi-temporal approach}

The multi-temporal approach deals with both inversion strategies, i.e. reduction of the number of unknown and increase of the number of measurement. The variations of surface geophysical parameters are at different temporal scales. In relatively short time window, one can assume that soil moisture is the main source of the time variation of backscatter. Thus, if one acquire sufficiently long-term data with high temporal resolution, e.g., scatterometer or scanSAR data, soil moisture processes can be estimated on the basis of the changes in radar measurements (Wagner et al., 1999; Wen and Su, 2003; Pathe et al., 2009) with reference backscattering values for dry and wet soil conditions as illustrated in Fig. 6. and Eq. (15) as:

$$
m_{R}(t)=\frac{\sigma^{0}(\mathrm{t})-\sigma_{d r y}^{0}}{\sigma_{w e t}^{0}-\sigma_{d r y}^{0}},
$$

where $m_{R}(\mathrm{t})$ is a relative measure of the surface soil moisture content. Although, in most of the previous studies, it has been limited to estimate a relative measure of soil moisture rather than absolute soil moisture contents (Kim and van Zyl, 2009), the multi-temporal approach has great potential for operational application through the use of currently orbiting radar systems.

\section{4) Fully polarimetric approach}

One of the most widely accepted way to address 
the inversion problem is to make use of multiple polarimetric measurements. By considering a coupled effect of roughness parameters and moisture contents on the polarimetric backscatter one can increase the performance of the inversion or geo-physical parameters. Studies on retrieving surface geophysical parameters using polarimetric SAR data have been focused on the use of co- and cross-polarized backscattering coefficients (Oh et al., 1992; Dubois et al., 1995; Haddad et al., 1996; Shi et al., 1997), such as:

$$
\left[\begin{array}{c}
m_{V} \\
s \\
l_{c}
\end{array}\right]=F^{-1}\left(\left[\begin{array}{c}
\sigma_{H H}^{0} \\
\sigma_{H V}^{0} \\
\sigma_{V V}^{0}
\end{array}\right]\right) .
$$

For example, one can obtain the surface geophysical parameters by inverting following set of equations (Oh et al., 1992):

$$
\begin{gathered}
\sigma_{H V}^{0}=0.11\left(m_{V}\right)^{0.7}(\cos \theta)^{2.2}\left[1-e^{-0.32(k s)^{1.8}}\right], \\
\sigma_{H H}^{0} / \sigma_{V V}^{0}=1-\left(\theta / 90^{\circ}\right)^{0.35 m \bar{V}^{0.65}} e^{-0.4(k s)^{1.4}}, \\
\sigma_{H V}^{0} / \sigma_{V V}^{0}=0.1\left(s / l_{c}+\sin 1.3 \theta\right)^{1.2}\left(1-e^{-0.9(k s)^{0.8}}\right) .
\end{gathered}
$$

In addition to the utilization of co- and crosspolarized backscatters, recent studies have reported the potential of the correlation coefficient between co-polarized channels as one of the surface roughness discriminator (Schuler et al., 2002; Hajnsek et al., 2003). The physical phenomenon inferred by the underlying sensitivity of the polarimetric coherence to the surface roughness is the depolarization of electromagnetic waves backscattered by a rough surface. Polarimetry deals with the full vector nature of electromagnetic waves, and, thus, the amount of information about a given scatterer is increased.

These studies have been extended in (Park et al., 2007, Park et al., 2009) to the utilization of the fully polarimetric signatures for retrieving geophysical parameters more accurately. The confounding influence of roughness statistics on the estimation of the soil moisture contents have been considered in the inversion algorithm that estimates volumetric moisture contents and roughness parameters simultaneously from the polarimetric alpha angle, the circular-polarization coherence, and the surface scattering component of the Pauli decomposition such as:

$$
\left[\begin{array}{c}
m_{V} \\
s \\
l_{c}
\end{array}\right]=F^{-1}\left(\left[\begin{array}{c}
\bar{\alpha} \\
\left|\rho_{R R L L}\right| \\
\left|S_{H H}+S_{V V}\right|^{2}
\end{array}\right]\right)
$$

where the function $\mathrm{F}$, in this case, can be modeled by the fully polarimetric IEM (Fung and Chen, 2010) or extended-Bragg model (Park et al., 2009). This approach seems to be a promising method to obtain geophysical parameters from space due to increasing availability of fully polarimetric space-borne imaging radar sensors. However, novel scattering models for polarimetric signatures need to be further improved and validated for diverse environmental conditions.

\section{Conclusion}

Radar remote sensing has great potential to provide soil moisture information, but it is very important to adequately characterize the compounding influence of roughness and vegetation cover on the soil moisture inversion process. The failure of the correct estimation of soil moisture can caused not from the lack of rigor of theoretical models but often from an unsuccessful parameterization of those geophysical properties of the target. Therefore, it is important to adequately characterize the compounding influence of different geophysical properties of the target for retrieving geophysical parameters more accurately.

In the inversion of the soil surfaces' geophysical parameters from radar measurement, it is required to obtain multiple measurements to avoid underdetermined problem. A number of approaches have been developed to deal with this issue. Particularly, with 
the rapidly increasing availability of space-borne multi-modal radar sensors, it is timely and important to investigate more accurate and operational algorithms capable of retrieving geophysical properties. It seems that the time-series approach combined with appropriate fully-polarimetric formulations on the temporal changes of geophysical parameters can provide the most promising results on soil moisture contents and their temporal evolution. It should be noted that, however, in developing a novel multi-modal inversion technique, one has to pay special attention to the response of the geophysical variables to the different types of radar measurements used in the inversion process. In particular, gathering multiple measurements becomes useless if the target system changes between successive observations or additional measurements involve new state variables.

\section{Acknowledgments}

This research is partially supported by a grant from Improvement of Independent Research Environment for Young Researchers program supported by the special coordination funds for Promoting Science and Technology, the Ministry of Education, Culture, Sports, Science and Technology, Japan.

\section{References}

Baghdadi, N., I. Gherboudj, M. Zirbi, M. Sahebi, C. King, and F. Bonn, 2004. Semiempirical calibration of the IEM backscattering model using radar images and moisture and roughness field measurements, International Journal of Remote Sensing, 25(18): 35933623.

Bass, F.G. and I.M. Fuks, 1979. Wave Scattering from Statistically Rough Surfaces, Oxford, Pergamon Press, Ltd. (International Series in Natural Philosophy. Volume 93).

Beckmann, P. and A. Spizzichino, 1963. The scattering of electromagnetic waves from rough surfaces, New York: Pergamon.

Bindlish, R. and A.P. Barros, 2000. Multifrequency soil moisture inversion from SAR measurements with the use of IEM, Remote Sensing of Environment, 71(1): 67-88.

Davidson, M.W.J., T. Le Toan, F. Mattia, G. Satalino, T. Manninen, and M. Borgeaud, 2000. On the characterization of agricultural soil roughness for radar remote sensing studies, IEEE Transactions on Geoscience and Remote Sensing, 38(2): 630-640.

Dobson, M.C., F.T. Ulaby, M.T. Hallikainen, and M.A. El-Rayes, 1985. Microwave dielectric behavior of wet soil-Part II: Dielectric mixing models, IEEE Transactions on Geoscience and Remote Sensing, GE-23(1): 35-46.

Dubois, P.C., J.J. van Zyl, and T. Engman, 1995. Measuring Soil Moisture with Imaging Radars, IEEE Transactions on Geoscience and Remote Sensing, 33(4): 916-926.

Fung, A.K., 1994. Microwave Scattering and Emission Models and Their Applications. Norwood, MA, Artech House.

Fung, A.K. and K.-S. Chen. 2010. Microwave Scattering and Emission Models for Users, Norwood, MA, Artech House.

Gade, M., W. Alper, C. Melsheimer, and G. Tanck, 2008. Classification of sediments on exposed tidal flats in the German Bight using multifrequency radar data, Remote Sensing of Environment, 112(4): 1603-1613.

Glenn, N.F. and J.R. Carr, 2003. The use of geostatistics in relating soil moisture to RADARSAT-1 SAR data obtained over the 
Great Basin, Nevada, USA, Computers and Geosciences, 29: 577-586.

Haddad, Z.S., P. Dubois, and J.J. van Zyl, 1996. Bayesian Estimation of Soil Parameters from Radar Backscatter Data, IEEE Transactions on Geoscience and Remote Sensing, 34(1): $76-82$.

Hajnsek, I., E. Pottier, and S.R. Cloude, 2003. Inversion of Surface Parameters from Polarimetric SAR, IEEE Transactions on Geoscience and Remote Sensing, 41(4): 727744.

Kim, Y. and J.J. van Zyl, 2009. Time-Series Approach to Estimate Soil Moisture Using Polarimetric Radar Data, IEEE Transactions on Geoscience and Remote Sensing, 47(8): 2519-2527.

Lee, J.S., D.L. Schuler, and T.L. Ainsworth, 2000. Polarimetric SAR data compensation for terrain azimuth slope variation, IEEE Transactions on Geoscience and Remote Sensing, 38(5): 2153-2163.

Lemaire, D., P. Sobieski, A. Guissard, and C. Craeye, 2002. Two-scale models for rough surface scattering : comparison between the Boundary Perturbation Method and the Integral Equation Method, Radio Science, 37(1): 1-16.

Oh, Y., K. Sarabandi, and F.T. Ulaby, 1992. An empirical model and an inversion technique for radar scattering from bare soil surfaces, IEEE Transactions on Geoscience and Remote Sensing, 30(2): 370-381.

Park, S.-E., J. Kim, W.M. Moon, and W.M. Boerner, 2007. Inversion of surface parameters from NASA/JPL AIRSAR polarimetric SAR data, Proc. of POLinSAR 2007, ESRIN, Frascati, Italy, Jan. 22-26.

Park, S.-E., L. Ferro-Famil, S. Allain, and E. Pottier, 2008. Analysis of Polarimetric Surface
Scattering in High Resolution SAR, Proc. of 2008 International Geoscience and Remote Sensing Symposium, Boston, USA, pp. 394397.

Park, S.-E., W.M. Moon, D. Kim, and J.-E. Kim, 2009. Estimation of surface roughness parameter in intertidal mudflat using airborne polarimetric SAR data, IEEE Transactions on Geoscience and Remote Sensing, 47(4): 10221031.

Pathe C., W. Wagner, D. Sabel, M. Doubkova, and J. Basara, 2009. Using ENVISAT ASAR Global Mode Data for Surface Soil Moisture Retrieval Over Oklahoma, USA, IEEE Transactions on Geoscience and Remote Sensing, 47(2): 468-480.

Quesney, A., S. Le H?garat-Mascle, O. Taconet, D. Vidal- Madjar, J.P. Wigneron, C. Loumagne, and M. Normand, 2000. Estimation of watershed soil moisture index from ERS/SAR data, Remote Sensing of Environment, 72(3): 290-303.

Rahman, M.M., M.S. Moran, D.P. Thoma, R. Bryant, C.D. Holifield Collins, T. Jackson, B.J. Orr, and M. Tischler, 2008. Mapping surface roughness and soil moisture using multi-angle radar imagery without ancillary data, Remote Sensing of Environment, 112(2): 391-402.

Shi, J., J. Wang, A.Y. Hsu, P.E. O’ Neill, and E.T. Engman, 1997. Estimation of bare surface soil moisture and surface roughness parameters using L-band SAR image data, IEEE Transactions on Geoscience and Remote Sensing, 35(5): 1254-1266.

Schuler, D.L., J.S. Lee, D. Kasilingam, and G. Nesti, 2002. Surface Roughness and Slope Measurements using Polarimetric SAR Data, IEEE Transactions on Geoscience and Remote Sensing, 40(3): 687-698. 
Ulaby, F.T., R.K. Moore, and A.K. Fung, 1982. Microwave Remote Sensing Volume II, Addison-Wesley, Reading, MA.

Ulaby F.T., 1998. SAR Biophysical Retrievals: Lessons learned and challenges to overcome, Proc. of 2nd Int. Workshop on Retrieval of Bio-\&Geo-Physical Parameters from SAR Data for Land Applications, ESTEC, The Netherlands, Oct. 21-23.

Ulaby, F.T. and C. Elachi, 1990. Radar polarimetry for geoscience applications, Artech House, Norwood, MA.

Wagner, W., G. Lemoine, and H. Rott, 1999. A method for estimating soil moisture from ERS scatterometer and soil data - Empirical data and model results, Remote Sensing of Environment, 70(2): 191-207.

Wang, J.R. and T.J. Schmugge, 1980. An empirical model for the complex dielectric permittivity of soils as a function of water content, IEEE Transactions on Geoscience and Remote Sensing, GE-18(4): 288-295.

Wen J. and Z. Su, 2003. A time series based method for estimating relative soil moisture with ERS wind scatterometer data. Geophysical Research Letter, 30.1397, doi:10.1029/2002 GL016557.

Wu, T.D., K.S. Chen, J. Shi, and A.K. Fung, 2001. A transition model for the reflection coefficient in surface scattering, IEEE Transactions on Geoscience and Remote Sensing, 39(9): 20402050.

Zribi, M. and M. Dechambre, 2002. A new empirical model to retrieve soil moisture and roughness from radar data, Remote Sensing of Environment, 84(1): 42-52. 